University of Wollongong

Research Online

Australian Institute for Innovative Materials -

Papers

Australian Institute for Innovative Materials

$1-1-2015$

Electrochemical synthesis of mesoporous Pt nanowires with highly electrocatalytic activity toward methanol oxidation reaction

Cuiling Liu

National Institute for Materials Science, Japan

Victor Malgras

National Institute for Materials Science, Japan, vm961@uowmail.edu.au

Saad Alshehri

King Saud University

Jung Ho Kim

University of Wollongong, jhk@uow.edu.au

Yusuke Yamauchi

National Institute for Materials Science, Japan

Follow this and additional works at: https://ro.uow.edu.au/aiimpapers

Part of the Engineering Commons, and the Physical Sciences and Mathematics Commons

Research Online is the open access institutional repository for the University of Wollongong. For further information contact the UOW Library: research-pubs@uow.edu.au 


\title{
Electrochemical synthesis of mesoporous Pt nanowires with highly electrocatalytic activity toward methanol oxidation reaction
}

\begin{abstract}
Self-supported one-dimensional (1D) mesoporous Pt nanowires (NWs) are prepared by confining micelle assembly in channels of a polycarbonate (PC) membrane. The obtained mesoporous Pt NWs show very high electrochemical activity and excellent durability as catalysts for methanol oxidation reaction (MOR) in comparison with the commercially available Pt black (PtB) catalyst. This work demonstrates that an appropriate combination of both self-supported 1D shape and mesoporous architecture indeed improve the electrocatalytic performances which is critical for further implementation and practical applications.
\end{abstract}

\section{Keywords}

pt, nanowires, highly, electrocatalytic, activity, toward, methanol, oxidation, reaction, synthesis, electrochemical, mesoporous

Disciplines

Engineering | Physical Sciences and Mathematics

\section{Publication Details}

Li, C., Malgras, V., Alshehri, S. M., Kim, J. \& Yamauchi, Y. (2015). Electrochemical synthesis of mesoporous Pt nanowires with highly electrocatalytic activity toward methanol oxidation reaction. Electrochimica Acta, 183 107-111. 


\title{
Electrochemical Synthesis of Mesoporous Pt Nanowires with Highly Electrocatalytic Activity toward Methanol Oxidation Reaction
}

\author{
Cuiling $\mathrm{Li}^{1}$, Victor Malgras ${ }^{1}$, Saad M. Alshehri ${ }^{2}$, Jung Ho Kim³ ${ }^{3}$ Yusuke Yamauchi*1
}

1 World Premier International (WPI) Research Center for Materials Nanoarchitectonics (MANA), National Institute for Materials Science (NIMS), 1-1 Namiki, Tsukuba, Ibaraki 305-0044 (Japan)

2 Department of Chemistry, College of Science, King Saud University, Riyadh 11451 (Saudi Arabia)

3 Institute for Superconducting and Electronic Materials (ISEM), Australian Institute for Innovative Materials (AIIM), University of Wollongong, North Wollongong, NSW 2500 (Australia)

$$
\text { E-mail: Yamauchi.Yusuke@nims.go.jp }
$$

\footnotetext{
Abstract: Self-supported one-dimensional (1D) mesoporous Pt nanowires (NWs) are prepared by utilizing micelle assembly in channels of a polycarbonate (PC) membrane. The obtained mesoporous Pt NWs show very high electrochemical activity and excellent durability as catalysts for methanol oxidation reaction (MOR), in comparison with the commercially available Pt black (PtB) catalyst. This work demonstrates that nice combination of both self-supported 1D shape and mesoporous architecture indeed improve the performance as electrocatalysts, which is an important finding for considering practical applications.
}

Keywords: Mesoporous materials; Pt catalysts; electrodeposition; surfactants 


\section{Introduction}

Reducing the usage of platinum (Pt) is a critical challenge for the commercial viability of fuel cells because of their high cost [1-3]. Improving the usage efficiency by minimizing the Pt content of the catalyst in fuel cells has been at the center of fundamental and technological concerns for the past decades [4-7]. From the standpoint of nanostructures, there are several effective approaches to produce Pt catalysts with large surface area (i.e., mesoporous Pt) [8-13] and to retain the initial active surface area for a long periods of time $[2,14-16]$.

Among several morphologies of Pt catalysts, self-supported 1D architecture has the advantage of long-term stability [17-20]. It is generally known that $\mathrm{Pt}$ catalysts lose their activities through oxidation/dissolution process, especially for supported materials (e.g., carbon-supported Pt catalysts) [21,22]. To overcome this issue, self-supported 1D NWs is a favorable solution for smooth electron transfer and to avoid structural collapse [23-25]. Recently, we have developed a novel 'electrochemical micelle assembly' soft-templating method which is a facile and effective route to obtain mesoporous metals with controlled pore sizes [18,26,27]. This micelle assembly allows good control over the shape of Pt-based materials, e.g. 0D nanoparticles, 1D NWs, and 2D films [18,26-28].

Mesoporous architectures are quite effective for increasing the electrochemically active area. Also, the concave surface on the mesopores can provide abundant catalytically active sites for the reactions, which is totally different from the convex surfaces usually observed in nanoparticles [26,29-31]. Recently, Fujita et al. have reported that the concave Au surfaces caused a higher catalytic activity toward CO oxidation [32]. Therefore, tailored synthesis of mesoporous Pt materials is important to further improve the Pt catalytic efficiency.

In this study, we successfully extend the micelle assembly approach [18] by triggering the growth of mesoporous Pt inside channels of polycarbonate (PC) membranes and prepare various mesoporous Pt NWs with different diameters and lengths. Our mesoporous Pt NWs have an ideal catalytic structure, which would both lead to high surface area and facile mass transportation through the mesopores inside the NWs. Our 1D mesoporous NWs show superior activity and stability when used as electrocatalyst in methanol oxidation 
reaction (MOR). 


\section{Experimental}

\subsection{Preparation for 1D mesoporous Pt NWs}

A thin Pt film (ca. $6 \mathrm{~nm}$ ) was deposited on one side of a commercially available PC membrane with $15 \mathrm{~nm}$ pore size. The PC membrane was then clamped between an ITO-glass slide and a section of glass tubing (30 $\mathrm{mm}$ in diameter). A platinum wire as a counter electrode and an $\mathrm{Ag} / \mathrm{AgCl}$ (saturated $\mathrm{KCl}$ ) reference electrode were included in the apparatus. The electrochemical deposition of 1D NWs was performed by using an electrochemical analyzer (CHI 842B, CH Instrument, U.S.) by applying a constant potential of -0.2 $\mathrm{V} v$ s. $\mathrm{Ag} / \mathrm{AgCl}$ at room temperature. The electrolyte solution used during the synthesis was $20 \mathrm{mM} \mathrm{K}_{2} \mathrm{PtCl}_{4}$ with 1.0 wt\% Brij58 $\left(\mathrm{C}_{16} \mathrm{H}_{33}\left(\mathrm{OCH}_{2} \mathrm{CH}_{2}\right)_{20} \mathrm{OH}\right)$. After the Pt deposition, the sample was immersed into a $5 \mathrm{M}$ $\mathrm{NaOH}$ aqueous solution and into an ethanol solution to remove the PC membrane and the surfactants, respectively

\subsection{Characterizations}

Scanning electron microscopy (SEM) images were obtained using a Hitachi HR-SEM SU8000 microscope with an accelerating voltage of $5 \mathrm{kV}$. Transmission electron microscope (TEM) and high-angle annular dark-field scanning TEM (HAADF-STEM) images were taken with a JEOL JEM-2100F microscope with an accelerating voltage of $200 \mathrm{kV}$. Wide-angle powder X-ray diffraction (XRD) profiles were recorded with a Rigaku Rint 2500 diffractometer with monochromated $\mathrm{Cu} \mathrm{Ka}$ radiation. X-ray photoelectronic spectroscopy (XPS) was carried out at room temperature by using a JPS-9010TR (JEOL) instrument with an Mg Ka X-ray source. All binding energies were calibrated by referencing to C $1 s(285.0 \mathrm{eV})$.

\subsection{Electrochemical analysis}

Cyclic voltammograms (CVs) and chronoamperometric measurements were achieved using a CHI 842B electrochemical analyzer (CH Instrument, USA). A conventional three-electrode cell was used, including an Ag/AgCl reference electrode, a platinum wire as a counter electrode, and a glassy carbon electrode (GCE, 3 $\mathrm{mm}$ in diameter) modified with the catalyst as a working electrode. Prior to coating the surface, the GCE 
was polished with 1.0 and $0.05 \mu \mathrm{m}$ alumina powder and rinsed with deionized water, followed with nitrogen gas drying. After being cleaned, $5.0 \mu \mathrm{g}$ of the samples was coated on the surface of GCE. Nafion solution (5.0 $\mu \mathrm{L}, 0.1 \mathrm{wt} \%$ ) was then coated on the surface and dried completely at room temperature. Prior to the measurements, we electrochemically cleaned the samples to completely remove the organic molecules. The applied conditions were as follows: potential range from -0.2 to $1.5 \mathrm{~V}$ (vs. $\mathrm{Ag} / \mathrm{AgCl}$ ) at a scan rate of 500 $\mathrm{mV} \mathrm{s}^{-1}$ for 100 cycles. The methanol oxidation reaction investigations were carried out in $0.5 \mathrm{M} \mathrm{H}_{2} \mathrm{SO}_{4}$ solution containing $0.5 \mathrm{M}$ methanol. All potential values were referenced to an $\mathrm{Ag} / \mathrm{AgCl}$ (saturated $\mathrm{KCl}$ ) electrode. 


\section{Results and Discussion}

The obtained NWs were characterized by scanning electron microscope (SEM) and transmission electron microscopy (TEM), as show in Figure 1. The SEM image at low magnification clearly shows well-defined 1D structures with no formation of irregularly shaped products, showing a high and uniform synthesis yield (> 99\%) (Figure 1a). The diameters of the obtained NWs have a narrow distribution, which is centered on $68 \mathrm{~nm}$ (Figure 1c). The diameter of the Pt NWs is slightly larger than the channel diameter of the used PC membrane. This is due to the non-uniformity of the PC channels. Similar situation has been often observed when using a PC membrane as a hard template $[18,33,34]$. On the surface of the obtained NWs, a large number of well-distributed mesopores can be observed, thus exposing the active Pt surfaces to the reagents. From the highly magnified SEM image (Figure 1b), the average pore size is estimated to be 6-7 nm, which is consistent with previously reported data on similar system [26]. The well-distributed mesoporous structures are further confirmed from the high-angle annular dark-field scanning TEM (HAADF-STEM) images (Figure 1d).

The sample was further investigated by high-resolution transmission electron microscopy (HRTEM) (Figure 2a-b). Generally, the electron beam cannot pass through mesoporous Pt sample due to its heavy weight, unlike mesoporous silica and carbon materials [35,36]. In the present case, the sample thickness is less than $100 \mathrm{~nm}$, so the HRTEM image can clearly show the lattice fringes which are randomly oriented inside the NWs (Figure 2b). The distance between two fringes is $0.23 \mathrm{~nm}$, which corresponds to Pt (111) crystal plane. The overall crystalline structures were further checked by wide-angle X-ray diffraction (XRD) measurement. The diffraction peaks can be assigned to a Pt fcc crystal (Figure 2c). By using the Scherrer equation, the crystallite size was roughly calculated from the Pt (111) diffraction peak to be $c a$. $5 \mathrm{~nm}$. The electronic state was also studied by using X-ray photoelectron spectroscopy (XPS). The peak positions coincide with the theoretical value of $\mathrm{Pt}^{0}$, meaning that the $\mathrm{Pt}$ species are completely reduced to metallic $\mathrm{Pt}$ under our experimental conditions (Figure 2d).

In our experimental methodology, Brij58 $\left(\mathrm{C}_{16} \mathrm{H}_{33}\left(\mathrm{OCH}_{2} \mathrm{CH}_{2}\right)_{20} \mathrm{OH}\right)$, a typical nonionic surfactants, is used as pore directing agent. When the concentration of Brij58 is higher than its critical micelle 
concentration (CMC, $0.08 \mathrm{mM}[18,26])$, spherical micelles with hydrophobic carbon chains in the interior and hydrophilic EO groups in the exterior are formed in the electrolyte. When the Pt species are added in the surfactant solution, the dissolved Pt ions coordinate with water molecules to form metal-aqua complexes. Through hydrogen bonding, the Pt-aqua complexes can then incorporate the exterior region of the micelles $[18,26]$. Thus, under optimized electrochemical conditions, the spherical micelles move towards the working electrode along with the dissolved Pt species [26]. Thus, 1D mesoporous Pt NWs (MPNWs) are subsequently obtained by confining the self-assembling micelles in the channels of the polycarbonate (PC) membrane. By changing the pore sizes of the PC membranes, 1D MPNWs with various diameters can be prepared. Herein, by changing the diameters of the PC membranes from $15 \mathrm{~nm}$ to $50 \mathrm{~nm}$ (Figure 3a and $\mathbf{3 b}$, respectively), the diameters of the obtained NWs can be correspondingly controlled without changing the mesoporous structure. The maximum length of the Pt NWs is of similar length to the thickness of the PC membrane. In order to avoid an overgrowth, the typical electrodeposition time was set to $600 \mathrm{~s}$. When this time was decreased down to 400 s or 200 s, NWs with different lengths were also obtained (Figure 4). In this case, the mesoporous Pt NWs have various aspect ratios, e.g., 38 (with a deposition time of 600 s), 25 (with a deposition time of $400 \mathrm{~s}$ ), and $\sim 13$ (with a deposition time of $200 \mathrm{~s}$ ). According to the previous reports, the catalytic activity of $1 \mathrm{D}$ constructions highly depends on the aspect ratios [37]. Therefore, the 1D MPNWs with the highest aspect ratios are selected and their electrocatalytic properties are further characterized.

The electrocatalytic performance (i.e., activity and stability) of our MPNWs toward methanol oxidation reaction (MOR) was evaluated and compared with a commercially available Pt black (abbreviated as PtB) catalyst (Figure 5a and 5b). The cyclic voltammograms (CVs) shown in Figure 5a exhibit the typical features of MOR on Pt catalysts, which possess two anodic peaks in the positive and negative sweeps. The 1D MPNWs showed superior activity with a mass-normalized current density of $192.8 \mathrm{~mA} \mathrm{mg}^{-1}$, which is around 2.7 times higher than that of the $\mathrm{PB}$ catalyst $\left(72.0 \mathrm{~mA} \mathrm{mg}{ }^{-1}\right)$. The higher activity of the $1 \mathrm{D}$ mesoporous Pt NWs can probably be ascribed to the sufficient amount of mesoporous structures which can be accessed by the reagents as well as to a higher electron transport efficiency due the 1D structure. It should 
be noticed that the activity of the present 1D mesoporous Pt NWs is similar to what was previously reported for 1D mesoporous Pt NWs with a diameter around $100 \mathrm{~nm}$ [18], indicating that all the pores inside the NWs are electrochemically available, regardless of their diameter. Such kind of 1D mesoporous architectures can make the whole Pt surface ready for catalytic reactions, which is a strong advantage for its practical applications. The durability of the samples toward MOR was also checked by chromoamperometric measurements. The typical plots shown in Figure 5c confirmed the superior stability of the 1D mesoporous Pt NWs by showing a slow current decay and a higher current density at every moment.

As higher stability was expected in the 1D mesoporous Pt NWs, the electrochemically active surface area (ECSA) retention after cycling treatment was further investigated for both samples. The cycling measurements were conducted in $0.5 \mathrm{M} \mathrm{H}_{2} \mathrm{SO}_{4}$ with a potential ranging from 0.6 to $1.0 \mathrm{~V}$, causing the surface oxidation/reduction cycles of the samples. The CV curves obtained after various periods were recorded, as shown in Figure 5d and e. A superior retention of the initial CV shape was confirmed for the 1D mesoporous Pt NWs in comparison to the PtB catalyst. The ECSA of each sample was estimated by integrating the charge passing in the hydrogen adsorption/desorption region, assuming that the charge required to oxidize a mono-layer of hydrogen on bright $\mathrm{Pt}$ is $210 \mu \mathrm{C} \mathrm{cm}^{-2}$ [18]. The initial ECSA of the 1D MPNWs (40.2 $\left.\mathrm{m}^{2} \mathrm{~g}^{-1}\right)$ is much higher than that of the PtB catalyst $\left(19.7 \mathrm{~m}^{2} \mathrm{~g}^{-1}\right)$. The loss of ECSA is inferior to $10 \%$ for the $1 \mathrm{D}$ MPNWs while the PtB catalyst show a loss of $12 \%$ (Figure 5f). More importantly, as shown in Figure 5b, our 1D MPNWs still show superior specific activity (ECSA normalized activity) toward MOR, indicating a much higher utilization efficiency of per unit Pt surface. This probably originates from the specific atomic Pt surfaces (e.g., kink and step sites) caused by the mesoporous structures $[10,11,18,26,38]$.

All these results are an evidence of the superior activity and stability of the self-supported 1D mesoporous Pt NWs. Without any doubt, the mesoporous structure is responsible for the superior activity due to the large amount of electrochemically active surfaces caused by the mesoporous structures. The self-assembled 1D morphology is also probably the main reason for the improved stability. Recently, Liang et al. report that free-standing Pt NW membranes exhibit remarkably higher durability than commercial 
supported and unsupported catalysts, which is crucial for polymer electrolyte membrane fuel cell (PEMFC) applications [14]. The important role of 1D morphology is under investigation.

\section{Conclusion}

We have successfully synthesized self-supported 1D mesoporous Pt NWs by a confined growth assisted by self-assembling micelles in a PC membrane. It was confirmed that the obtained Pt nanowires have a uniform mesoporous structure. During the electrochemical measurements, the 1D mesoporous Pt NWs showed superior electrocatalytic activity and stability. This performance is ascribed to the combination of a 1D morphology and a mesoporous architecture. The electrochemical deposition approach allows a fine control over the diameters and the lengths of the nanowires. Our electrochemical approach is widely applicable to other metal and alloy systems. A recent report from Wang et al. has demonstrated that multilayered mesoporous Pt/Pd bimetallic alternating films are prepared by using layer-by-layer electrochemical deposition [26]. By further extending this technique, we can prepare multilayered mesoporous metal NWs, which will bring out new properties in future.

\section{Acknowledgement}

The authors extend their appreciation to the Deanship of Scientific Research support from the Visiting Professor Program of King Saud University to undertake Research in Saudi Arabia. 
Figure 1

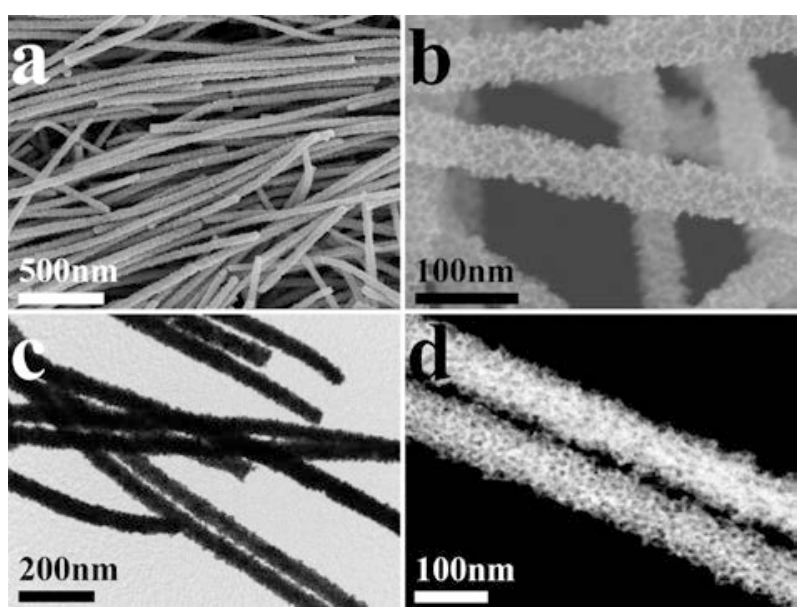

Figure 1. a) Low- and b) high-magnification SEM images, c) TEM, and d) HAADF-STEM images of 1D MPNWs obtained by using a deposition time of $600 \mathrm{~s}$. 
Figure 2
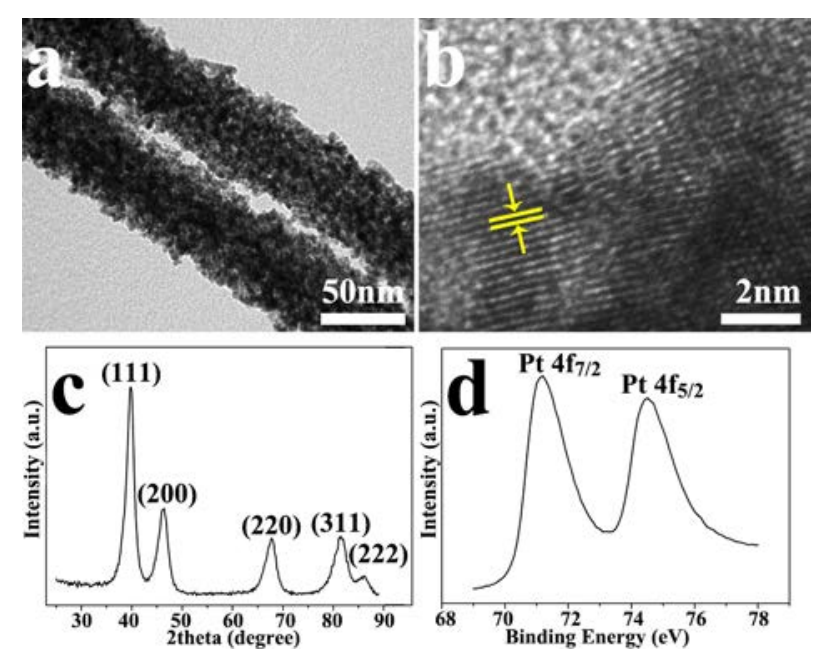

Figure 2. a) Low-magnification and b) high resolution TEM images of the 1D MPNWs c) Wide-angle XRD profile and d) high resolution XPS centered on Pt 4f obtained from the typical 1D MPNWs sample prepared with a deposition time of $600 \mathrm{~s}$. 


\section{Figure 3}
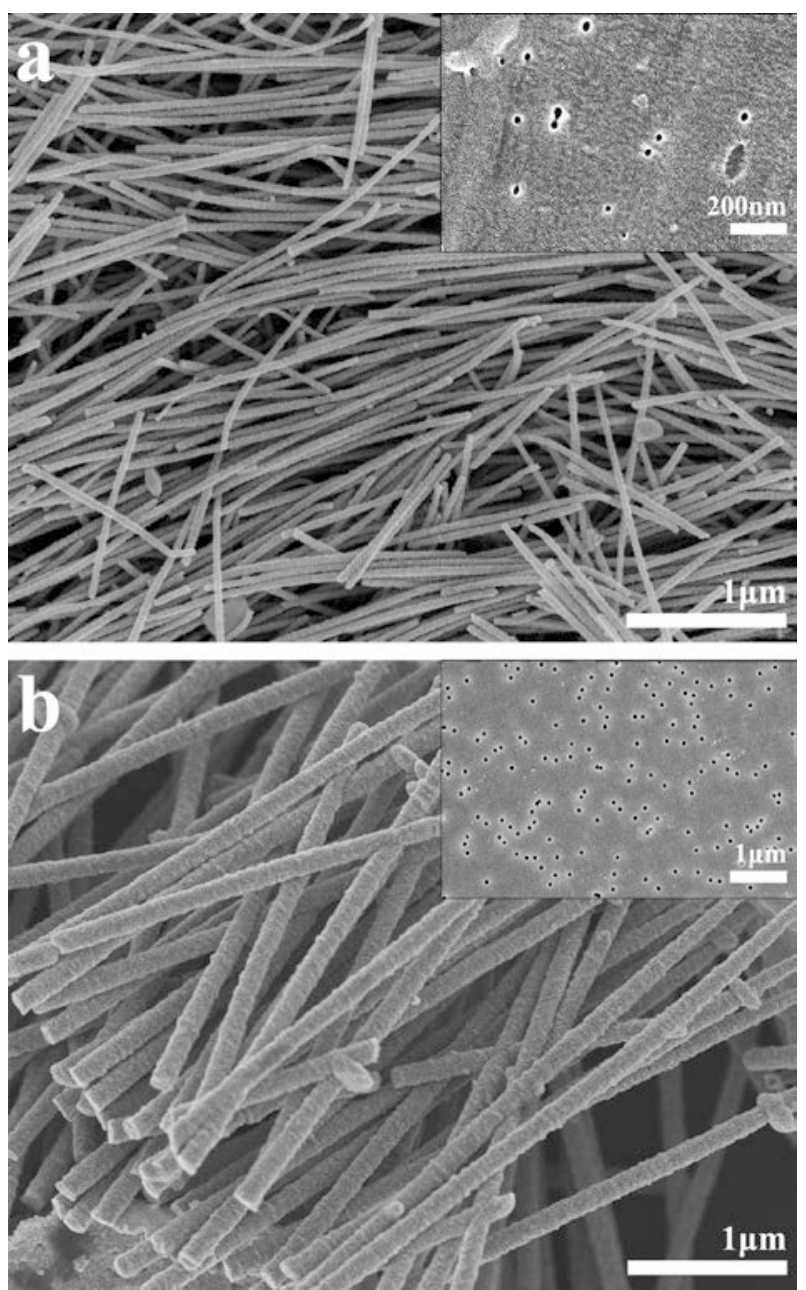

Figure 3. Typical SEM images of the 1D MPNWs deposited using PC membranes with different pore sizes: a) $15 \mathrm{~nm}$, and b) $50 \mathrm{~nm}$. The right-top inset image in each panel is the SEM images of the PC membranes used for the electrodeposition. 
Figure 4
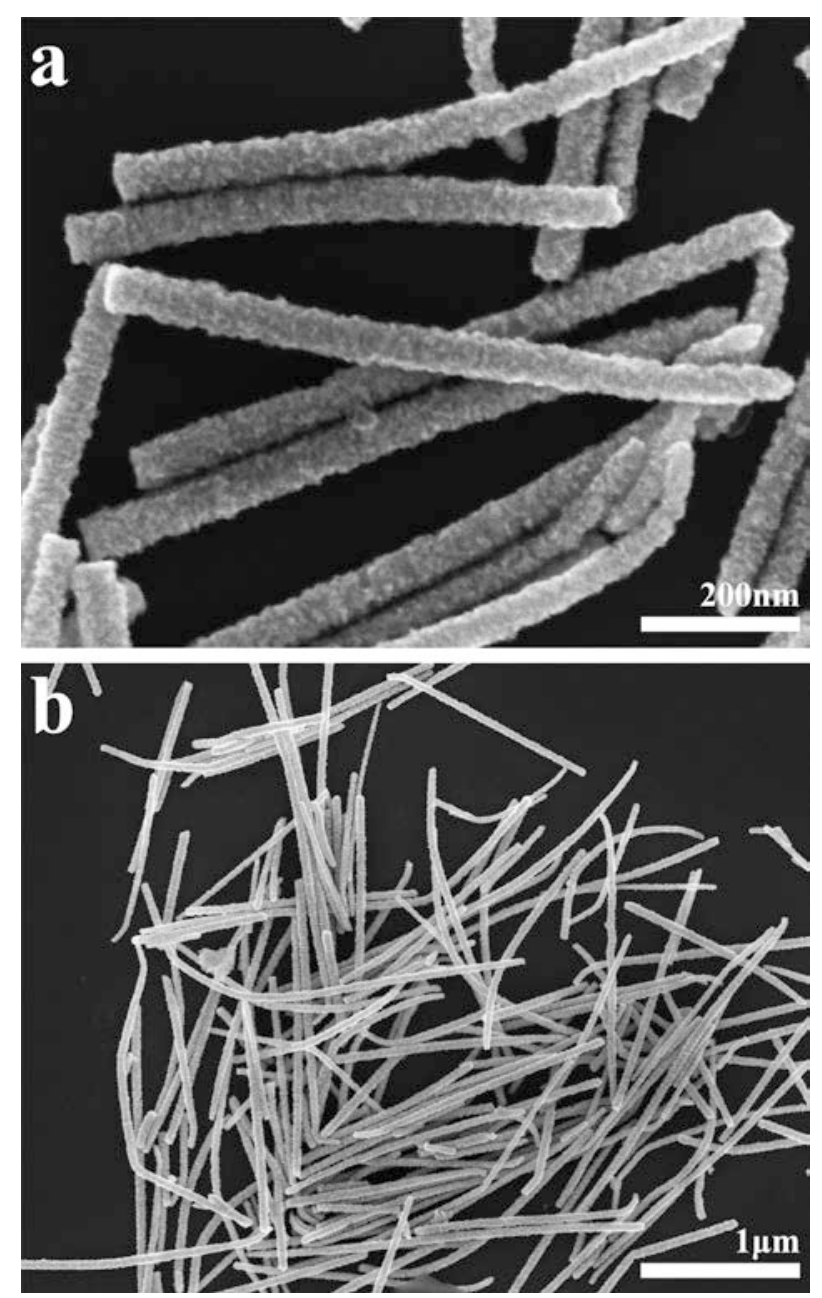

Figure 4. SEM images of the 1D MPNWs prepared by changing the deposition time: a) $200 \mathrm{~s}$, and b) $400 \mathrm{~s}$. 
Figure 5
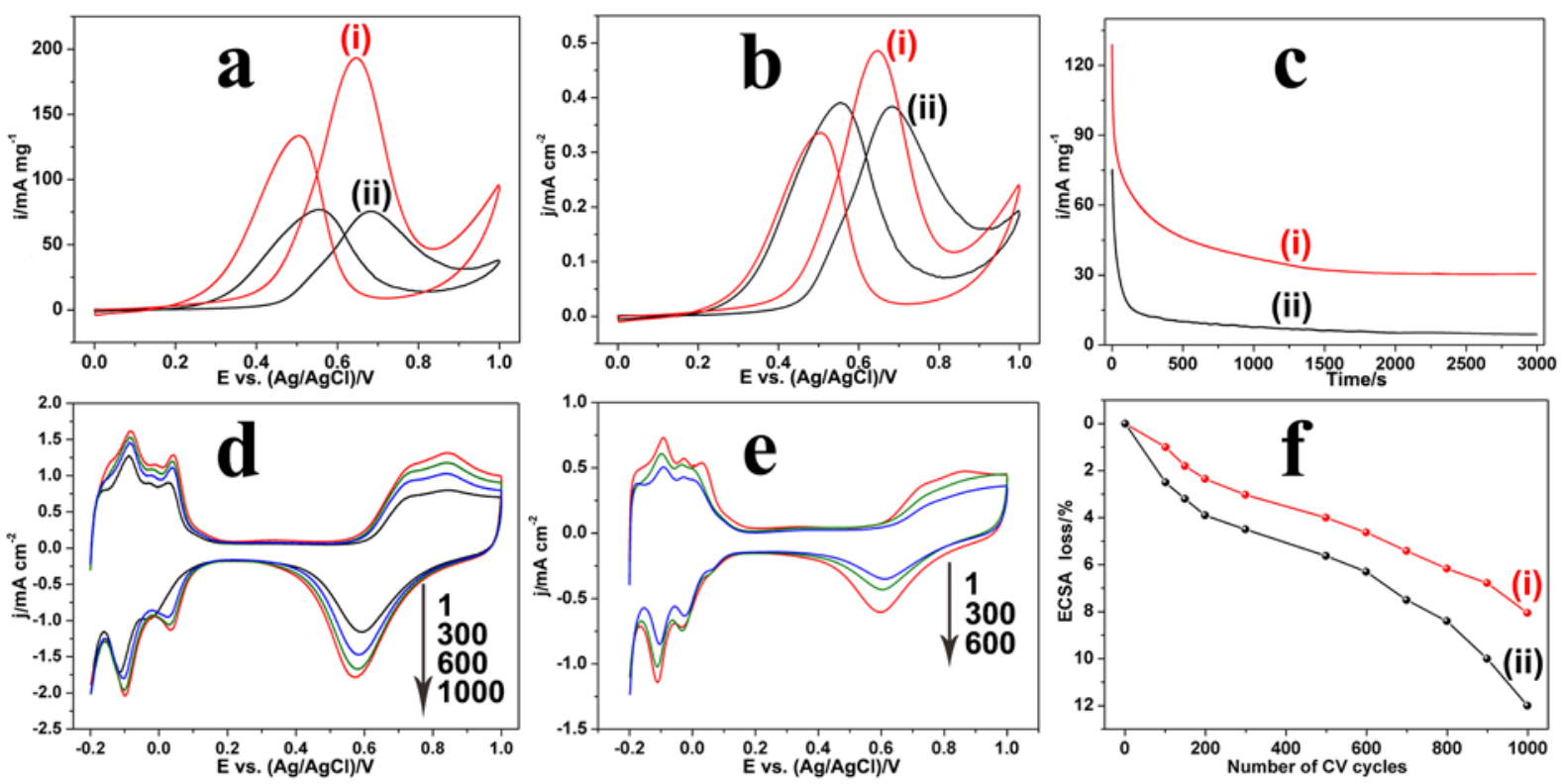

Figure 5. (a, b) Cyclic voltammograms (CVs) and (c) chronoamperometric plots of the 1D MPNWs (i) and the commercially available PtB catalyst (ii) toward methanol oxidation reaction. The CVs were recorded in an electrolyte of $0.5 \mathrm{M} \mathrm{H}_{2} \mathrm{SO}_{4}$ containing $0.5 \mathrm{M} \mathrm{CH} 3 \mathrm{OH}$ at a scan rate of $50 \mathrm{mV} \mathrm{s}^{-1}$. The $i$ - $t$ plots were obtained with the same electrolyte under a constant potential of $0.6 \mathrm{~V}$. The currents were normalized by the (a, c) Pt mass amount and (b) Pt ECSA. (d, e) Comparison of electrochemical durability of (d) 1D MPNWs and e) PtB catalyst after 1, 300, 600 and 1000 cycles. (f) ECSA loss of 1D MPNWs (i) and PtB (ii) catalyst with increasing the number of CV cycles. 


\section{References}

[1] Y. Bing, H. Liu, L. Zhang, D. Ghosh, J. Zhang, Chem. Soc. Rev. 2010, 39, 2184-2202.

[2] B. Lim, M. Jiang, P. H. C. Camargo, E. C. Cho, J. Tao, X. Lu, Y. Zhu, Y. Xia, Science 2009, 324, 1302-1305.

[3] C. Li, B. Jiang, M. Imura, V. Malgras, Y. Yamauchi, Chem. Commun. 2014, 50, 15337-15340.

[4] C. Wang, M. Chi, D. Li, D. Strmcnik, D. van der Vliet, G. Wang, V. Komanicky, K.-C. Chang, A. P. Paulikas, D. Tripkovic, J. Pearson, K. L. More, N. M. Markovic, V. R. Stamenkovic, J. Am. Chem. Soc. 2011, 133, 14396-14403.

[5] G. Wang, B. Huang, L. Xiao, Z. Ren, H. Chen, D. Wang, H. D. Abruña, J. Lu, L. Zhuang, J. Am. Chem. Soc. 2014, 136, 9643-9649.

[6] K. Sasaki, H. Naohara, Y. Cai, Y. M. Choi, P. Liu, M. B. Vukmirovic, J. X. Wang, R. R. Adzic, Angew. Chem. Int. Ed. 2010, 49, 8602-8607.

[7] X. Huang, E. Zhu, Y. Chen, Y. Li, C.-Y. Chiu, Y. Xu, Z. Lin, X. Duan, Y. Huang, Adv. Mater. 2013, 25,2974-2979.

[8] H. Wang, H. Y. Jeong, M. Imura, L. Wang, L. Radhakrishnan, N. Fujita, T. Castle, O. Terasaki, Y. Yamauchi, J. Am. Chem. Soc. 2011, 133, 14526-14529.

[9] K.-S. Choi, E. W. McFarland, G. D. Stucky, Adv. Mater. 2003, 15, 2018-2021.

[10] L. Wang, Y. Yamauchi, J. Am. Chem. Soc. 2009, 131, 9152-9153.

[11] H. Ataee-Esfahani, M. Imura, Y. Yamauchi, Angew. Chem. Int. Ed. 2013, 52, 13611-13615.

[12] X. Teng, X. Liang, S. Maksimuk, H. Yang, Small 2006, 2, 249-253.

[13] S. Yang, X. Luo, Nanoscale 2014, 6, 4438-4457.

[14] H.-W. Liang, X. Cao, F. Zhou, C.-H. Cui, W.-J. Zhang, S.-H. Yu, Adv. Mater. 2011, 23, 1467-1471.

[15] B. Y. Xia, W. T. Ng. H. B. Wu, X. Wang, X. W. Lou, Angew. Chem. Int. Ed. 2012, 51, 7213-7216.

[16] C. Li, M. Imura, Y. Yamauchi, Chem. Eur. J. 2014, 20, 3277-3282.

[17] L. Liu, E. Pippel, R. Scholz, U. Gösele, Nano Lett. 2009, 9, 4352-4358.

[18] C. Li, T. Sato, Y. Yamauchi, Angew. Chem. Int. Ed. 2013, 52, 8050-8053. 
[19] S. M. Alia, G. Zhang, D. Kisailus, D. Li, S. Gu, K. Jensen, Y. Yan, Adv. Funct. Mater. 2010, 20, 3742-3746.

[20] Z. Chen, M. Waje, W. Li, Y. Yan, Angew. Chem. Int. Ed. 2007, 46, 4060-4063.

[21] J. Wei, H. Wang, Y. Deng, Z. Sun, L. Shi, B. Tu, M. Luqman, D. Zhao, J. Am. Chem. Soc. 2011, 133, 20369-20377.

[22] Y.-P. Chen, C.-T. Chen, Y. Hung, C.-M. Chou, T.-P. Liu, M.-R. Liang, C.-T. Chen, C.-Y. Mou, J. Am. Chem. Soc. 2013, 135, 1516-1523.

[23] C. Koenigsmann, W.-P. Zhou, R. R. Adzic, E. Sutter, S. S. Wong, Nano Lett. 2010, 10, 2806-2811.

[24] H.-H. Li, S. Zhao, M. gong, C.-H. Cui, D. He, H.-W. Liang, L. Wu, S.-H. Yu, Angew. Chem. Int. Ed. 2013, 52, 7472-7476.

[25] C. Wang, M. Waje, X. Wang, J. M. Tang, R. C. Haddon, Y. Yan, Nano Lett. 2004, 4, 345-348.

[26] H. Wang, L. Wang, T. Sato, Y. Sakamoto, S. Tominaka, K. Miyasaka, N. Miyamoto, Y. Nemoto, O. Terasaki, Y. Yamauchi, Chem. Mater. 2012, 24, 1591-1598.

[27] H. Wang, S. Ishihara, K. Ariga, Y. Yamauchi, J. Am. Chem. Soc. 2012, 134, 10819-10821.

[28] C. Li, M. Imura, Y. Yamauchi, Phys. Chem. Chem. Phys. 2014, 16, 8787-8790.

[29] E. A. Franceschini, M. M. Bruno, F. A. Viva, F. J. Williams, M. Jobbágy, H. R. Corti, Electrochim. Acta 2012, 71, 173-180.

[30] A. Kucernak, J. Jiang, Chem. Eng. J. 2003, 93, 81-90.

[31] J. Jiang, A. Kucernak, J. Electroanal. Chem., 2002, 520, 64-70.

[32] T. Fujita, P. Guan, K. McKenna, X. Lang, A. Hirata, L. Zhang, T. Tokunaga, S. Arai, Y. Yamamoto, N. Tanaka, Y. Ishikawa, N. Asao, Y. Yamamoto, J. Erlebacher, M. Chen, Nature Mater. 2012, 11, 775-780.

[33] N. Suzuki, T. Kimura, Y. Yamauchi, J. mater. Chem. 2010, 20, 5294-5300.

[34] H. Zhou, S. S. Wong, ACS Nano 2008, 2, 944-958.

[35] J. Wei, H. Wang, Y. Deng, Z. Sun, L. Shi, B. Tu, M. Luqman, D. Zhao, J. Am. Chem. Soc. 2011, 133, 20369-20377.

[36] J. Tang, J. Liu, C. Li, Y. Li, M. O. Tade, S. Dai, Y. Yamauchi, Angew. Chem. Int. Ed. 2015, 54, 588-593. 
[37] P. Wu, H. Zhang, Y. Qian, Y. Hu, H. Zhang, C. Cai, J. Phys. Chem. C 2013, 117, 19091-19100.

[38] C. Li, V. Malgras, A. Aldalbahi, Y. Yamauchi, Chem. Asian J. 2015, 10, 316-320. 